\title{
Mellin analysis of partial differential equations in papers of $\mathrm{B}$. Ziemian
}

\author{
by Henryk KoŁakowski (Warszawa)
}

\begin{abstract}
Existence and regularity theorems for Fuchsian type differential operators and the theory of second microlocalization are presented.

1. Introduction. The study of solutions to singular elliptic partial differential equations is quite different from that of solutions to elliptic equations. In the latter case solutions are completely controlled by the behaviour of their Fourier transforms at infinity (first wave front set). In the case of corner equations the Fourier transform is replaced by the multidimensional Mellin transform. Using special spaces of distributions (subspaces of Mellin transformable distributions) one can measure local regularity of solutions to such equations.

Let $a, b \in \mathbb{R}^{n}, a=\left(a_{1}, \ldots, a_{n}\right), b=\left(b_{1}, \ldots, b_{n}\right)$. Then $a<b$ denotes $a_{j}<b_{j}$ for $j=1, \ldots, n$. Let $\mathbb{R}_{+}^{n}=\left\{x \in \mathbb{R}^{n}: x>0\right\}, I=(0, \mathbf{r}]=\left\{x \in \mathbb{R}_{+}^{n}:\right.$ $x \leq \mathbf{r}\}, \mathbf{r}=(r, \ldots, r) \in \mathbb{R}_{+}^{n}, \mathbf{r}<\mathbf{1}, \mathbf{1}=(1, \ldots, 1)$. We write

$$
\frac{\partial}{\partial x}=\left(\frac{\partial}{\partial x_{1}}, \ldots, \frac{\partial}{\partial x_{n}}\right)=\left(D_{1}, \ldots, D_{n}\right), \quad x \frac{\partial}{\partial x}=\left(x_{1} \frac{\partial}{\partial x_{1}}, \ldots, x_{n} \frac{\partial}{\partial x_{n}}\right) .
$$
\end{abstract}

For $y \in \mathbb{R}^{n}$ we define $\mu(y)=\left(e^{-y_{1}}, \ldots, e^{-y_{n}}\right)$.

Let $a \in \mathbb{R}^{n}$. We denote by $M_{a}=M_{a}(I)$ the complex vector space of functions $\varphi \in C^{\infty}(I)$ such that for $\nu \in \mathbb{N}_{0}^{n}$,

$$
\varrho_{a, \nu}(\varphi)=\sup _{x \in I}\left|x^{a+\nu+1} D^{\nu} \varphi(x)\right|<\infty
$$

with the topology given by the seminorms $\varrho_{a, \nu}$. The space $M_{(\omega)}=M_{(\omega)}(I)$ for $\omega \in(\mathbb{R} \cup\{\infty\})^{n}$ is the inductive limit

$$
M_{(\omega)}=\underset{a<\omega}{\lim _{a}} M_{a}
$$

2000 Mathematics Subject Classification: 35A07, 35A20, 35A27.

Key words and phrases: Mellin transformation, singular elliptic operators, propagation of singularities. 
and $M_{(\omega)}^{\prime}$ is the dual of $M_{(\omega)}$. The space $M^{\prime}=\bigcup_{\omega} M_{(\omega)}^{\prime} \subset \mathcal{D}^{\prime}\left(\mathbb{R}_{+}^{n}\right)$ is called the space of Mellin (transformable) distributions on $I$. If $u \in M_{(\omega)}^{\prime}$ then the Mellin transform of $u$ is defined by

$$
\mathcal{M} u(z)=u\left[x^{-z-1}\right] \quad \text { for } z \in \mathbb{C}^{n}, \operatorname{Re} z<\omega .
$$

Since $\mathcal{M}\left(x \frac{\partial}{\partial x} u\right)(z)=z \mathcal{M} u(z)$ the Mellin transformation is well suited for studying Fuchsian type differential operators of the form $R=R(x, x \partial / \partial x)$ where $R(x, z)$ is a polynomial of degree $m$ in $z \in \mathbb{C}^{n}$. The space of Mellin distributions on $\mathbb{R}_{+}^{n}$ for every $\alpha \in \mathbb{R}^{n}$ is defined as the dual $\mathfrak{M}_{\alpha}^{\prime}$ of the space

$$
\mathfrak{M}_{\alpha}=\mathfrak{M}_{\alpha}\left(\mathbb{R}_{+}^{n}\right)=\left\{\sigma \in C^{\infty}\left(\mathbb{R}_{+}^{n}\right):\left(x^{\alpha+1} \sigma\right) \circ \mu \in S\left(\mathbb{R}^{n}\right)\right\}
$$

with the topology induced from the space $S\left(\mathbb{R}^{n}\right)$. The Fourier-Mellin transform of $u \in \mathfrak{M}_{\alpha}^{\prime}$ is the distribution

$$
\mathcal{M}_{\alpha} u=F^{-1}\left(e^{\alpha s}(u \circ \mu)\right) \in S^{\prime}\left(\mathbb{R}^{n}\right)
$$

where the inverse Fourier transform $F^{-1}$ is defined as

$$
F^{-1} \psi(\xi)=\int_{\mathbb{R}^{n}} e^{i s \xi} \psi(s) d s \quad \text { for } \psi \in S\left(\mathbb{R}^{n}\right) .
$$

2. Local existence and regularity theorems. Let $R$ be a second order partial differential operator on an open set $0 \in U \subset \mathbb{R}^{2}$,

$$
R=\sum_{|\nu| \leq 2} a_{\nu}(x)\left(x_{1} \frac{\partial}{\partial x_{1}}\right)^{\nu_{1}}\left(x_{2} \frac{\partial}{\partial x_{2}}\right)^{\nu_{2}}
$$

where $a_{\nu}$ are smooth functions on $U$ and

1) $R$ has a regular singularity at zero: $a_{20}(0) \cdot a_{02}(0) \neq 0, a_{\nu}(0)=0$ otherwise,

2) $R$ is degenerate elliptic in $U: a_{20}(x) \cdot a_{02}(x)>0$ for $x \in U$.

Theorem 1 (Z. Szmydt and B. Ziemian [2]). Let $w \in \mathcal{D}^{\prime}(U)$ be of order $\left(s_{1}, s_{2}\right)$ about zero (i.e. $w=D_{1}^{s_{1}} D_{2}^{s_{2}} f, f$ continuous on $U$ ). Then for every proper subcone $\Gamma \subset \mathbb{R}_{+}^{2}$ and $\alpha=\left(\alpha_{1}, \alpha_{2}\right) \in \mathbb{R}^{2}$ such that $\alpha_{1}+\alpha_{2}<-s_{1}-$ $s_{2}$ there exists a neighbourhood $V$ of zero in $\mathbb{R}^{2}$ and a Mellin distribution $u \in \mathfrak{M}_{\alpha}^{\prime}$ such that

$$
R u=w \quad \text { on } \Gamma \cap V .
$$

In [3], the cone $\Gamma$ is replaced by a set $\Gamma_{\varrho} \subset \mathbb{R}_{+}^{2}, \varrho=\left(\varrho_{1}, \varrho_{2}\right) \in \mathbb{R}_{+}^{2}$, $\varrho_{1}<1<\varrho_{2}$, which is tangent to $\left\{x_{1}=0\right\}$ of order $1 / \varrho_{1}-1$ and to $\left\{x_{2}=0\right\}$ of order $\varrho_{2}-1$ (explicitly $\Gamma_{\varrho}=\left\{\left(x_{1}, x_{2}\right): x_{1}>0, x_{1}^{\varrho_{2}}<x_{2}<x_{1}^{\varrho_{1}}\right\}$, and $R$ is a differential operator as in Theorem 1 .

Theorem 2 (Z. Szmydt and B. Ziemian [3]). Let $w \in \mathcal{D}^{\prime}(U)$ be of order $\left(s_{1}, s_{2}\right)$ about zero. Then for every $\Gamma_{\varrho} \subset \mathbb{R}_{+}^{2}$ and $\alpha=\left(\alpha_{1}, \alpha_{2}\right) \in \mathbb{R}^{2}$ such 
that

$$
\alpha_{1}+\varrho_{2} \alpha_{2}<-s_{1}-\varrho_{1} s_{2}, \quad \alpha_{1}+\varrho_{2} \alpha_{2}<-s_{1}-\varrho_{2} s_{2}
$$

there exists a neighbourhood $V$ of zero in $\mathbb{R}^{2}$ and a Mellin distribution $u \in \mathfrak{M}_{\alpha}^{\prime}$ such that

$$
R u=w \quad \text { on } \Gamma_{\varrho} \cap V .
$$

A continuation of [2] and [3] is [5].

Denote by $M_{(\omega)}^{\prime s}$ the subspace of $u \in M_{(\omega)}^{\prime}$ such that $\mathcal{M} u(z)$ is $O\left(\prod_{j=1}^{n}\left(1+\left|\operatorname{Im} z_{j}\right|\right)^{s}\right)$ as $|\operatorname{Im} z| \rightarrow+\infty$ locally uniformly in $\{\operatorname{Re} z=x<\omega\}$. Let $\Omega \subset \mathbb{R}_{+}^{n-1}$ be an arbitrary open bounded set and $\Gamma_{K}(K=\bar{\Omega})$ be the "curved cone": $\Gamma_{K}=\psi\left(\mathbb{R}_{+}^{1} \times K\right)$ where

$$
\psi: \mathbb{R}_{+}^{1} \times K \ni(t, \varrho) \mapsto x=\left(t, t^{\varrho_{1}}, \ldots, t^{\varrho_{n-1}}\right) \in \mathbb{R}_{+}^{n} .
$$

Let $u \in M^{\prime}$. We say that $u \in M_{(\omega)}^{\prime s} \Gamma_{K^{-}}$locally at zero if there exists a cutoff function $\kappa$ subordinate to $\Gamma_{K}$ such that $\kappa u \in M_{(\omega)}^{\prime s}$. Let $R=R(x, x \partial / \partial x)$ where $R(x, z)$ is a polynomial of degree $m$ in $z$ with smooth coefficients on $U, P(z)=R(0, z)$ (the principal Mellin symbol of $R$ at zero). By assumption

$$
\begin{aligned}
R & =P\left(x \frac{\partial}{\partial x}\right)-Q\left(x, x \frac{\partial}{\partial x}\right), \\
Q\left(x, x \frac{\partial}{\partial x}\right) & =x_{1} Q_{1}\left(x, x \frac{\partial}{\partial x}\right)+\ldots+x_{n} Q_{n}\left(x, x \frac{\partial}{\partial x}\right),
\end{aligned}
$$

where $Q_{1}, \ldots, Q_{n}$ are differential operators of order $m$ and for every $\alpha \in \mathbb{R}^{n}$ there exist $A$ and $c_{0}$ such that (ellipticity condition)

$$
|P(\alpha+i \beta)|>c_{0} \prod_{j=1}^{n}\left(1+\left|\beta_{j}\right|\right)^{m} \quad \text { for }|\beta| \geq A .
$$

For $\alpha \in \mathbb{R}^{n}$ define $l(\alpha, \varrho)=\alpha_{1}+\varrho_{1} \alpha_{2}+\ldots+\varrho_{n-1} \alpha_{n}$ for $\varrho \in K$.

Theorem 3 (Z. Szmydt and B. Ziemian [4], [5]). Suppose $w \in M_{(\omega)}^{\prime s}$, where $\omega \in \mathbb{R}^{n}, s \in \mathbb{R}$ and $\max _{\varrho \in K} l(\alpha-\omega, \varrho)<0$. Then there exist $u \in M_{(\alpha)}^{\prime s-m} \Gamma_{K}$-locally and an open neighbourhood $W$ of zero in $\mathbb{R}^{n}$ such that

$$
R u=w \quad \text { on } \Gamma_{\Omega} \cap W .
$$

Regularity of solutions to degenerate elliptic operators is also studied in [6] and [7]. Such operators were studied e.g. by M. Kashiwara and R. Melrose. These operators are 2-microelliptic in direction $\delta x \in \mathbb{R}_{+}^{n}$ (cf. [8]).

Let $\Omega^{1}$ be an open subset of $\mathbb{C}$ such that together with every $\zeta \in \Omega^{1}$ it also contains the half-line $\zeta+\mathbb{R}_{-}\left(\Omega^{1}\right.$ is an $\mathbb{R}_{-}$-connected open subset of $\left.\mathbb{C}\right)$ such that for every $\zeta \in \Omega^{1}$ the set of points not in $\Omega^{1}$ whose real part is less 
than $\operatorname{Re} \zeta$ is compact. In applications $\Omega^{1}=\mathbb{C} \backslash \bigcup_{j=1}^{k} L_{j}$ where $L_{j}=\xi_{j}+\mathbb{R}_{+}$ for some $\xi_{1}, \ldots, \xi_{k}$. Let $\zeta=A z$, where

$$
\zeta_{1}=z_{1}+\ldots+z_{n}, \quad \zeta_{j}=z_{j} \quad \text { for } j=2, \ldots, n \text {. }
$$

Set $\Omega=A^{-1}\left(\Omega^{1} \times \mathbb{C}^{n-1}\right)$ and let $s \in \mathbb{R}$. We say that a Mellin distribution $u$ with support in a proper cone $\Gamma \subset \mathbb{R}_{+}^{n}$ belongs to $M(\Omega ; s)$ if $\mathcal{M} u$ is holomorphic in $\Omega$ and for any open neighbourhood $W$ of $\mathbb{C} \backslash \Omega^{1}$,

$$
\left|\left(\mathcal{M} \circ A^{-1}\right)(\alpha+i \beta)\right| \leq C(1+|\beta|)^{s} \quad \text { for } \alpha+i \beta \in \Lambda=(\mathbb{C} \backslash W) \times \mathbb{C}^{n-1}
$$

where $C=C(W, \alpha)$ is locally bounded in $\alpha \in \operatorname{Re} \Lambda$. Let $P$ be a polynomial in $\mathbb{C}^{n}$, and $\widetilde{\alpha} \in \mathbb{R}^{n}$. Set $\widetilde{a}=A \widetilde{\alpha}$ and $\mathcal{P}(\zeta)=P \circ A^{-1}(\zeta)$, and let $(1 / \mathcal{P})_{\tilde{a}}$ be a regularization to a distribution in $S^{\prime}\left(\mathbb{R}^{n}\right)$ of the function $b \mapsto(1 / \mathcal{P})(\widetilde{a}+i b)$, $\zeta=\left(\zeta_{1}, \zeta^{\prime}\right), \zeta^{\prime} \in \mathbb{C}^{n-1}$. Let $\Omega^{1} \subset \mathbb{C}$ be the largest subset such that the function

$\zeta \mapsto \widetilde{\mathcal{C}}(\zeta)=(2 \pi)^{-n}(1 / \mathcal{P})_{\tilde{a}}[F(a+i \gamma) K(\zeta-\widetilde{a}-i \gamma)] \quad$ for $\operatorname{Re} \zeta_{1}<\widetilde{a}_{1}, \zeta^{\prime} \in \mathbb{C}^{n-1}$ extends to a holomorphic function on $\left(\Omega^{1} \cap \widetilde{\Omega}^{1}\right) \times \mathbb{C}^{n-1}$; here $F$ is a holomorphic function on $\widetilde{\Omega}^{1}$ such that for any open neighbourhood $W$ of $\mathbb{C} \backslash \widetilde{\Omega}^{1}$ there exist constants $C$ and $M$ such that

$$
|F(a+i b)| \leq C(1+|b|)^{M} \quad \text { on }(\mathbb{C} \backslash W) \times \mathbb{C}^{n-1}
$$

locally uniformly in $a \in \operatorname{Re} \widetilde{\Omega}^{1} \times \mathbb{R}^{n-1}$, and $\widetilde{\Omega}^{1}$ is an open subset of $\mathbb{C}$ with the same properties as $\Omega^{1}, K(\zeta)=(\mathcal{M} \kappa) \circ A^{-1}(\zeta), \kappa=\varphi \cdot \widetilde{\kappa}$ is a conical cut-off function, i.e. $\varphi$ is a test function, $\varphi \equiv 1$ near the origin and $\widetilde{\kappa} \in C^{\infty}\left(\mathbb{R}_{+}^{n}\right)$ is homogeneous of order 0 , supported by a proper subcone of $\mathbb{R}_{+}^{n}$. The radial characteristic set $\operatorname{Char}_{\widetilde{\alpha}} P$ is defined by

$$
\operatorname{Char}_{\widetilde{\alpha}} P=\mathbb{C}^{n} \backslash A^{-1}\left(\Omega^{1} \times \mathbb{C}^{n-1}\right) .
$$

This set depends on the geometry of the zero set $\{P(z)=0\}$. If $P$ is a polynomial in one variable $z \in \mathbb{C}$, then for $\alpha \in \mathbb{R}$, $\operatorname{Char}_{\alpha} P=\{z \in \mathbb{C}$ : $P(z)=0, \operatorname{Re} z \geq \alpha\}$.

Let $\delta x_{0} \in \mathbb{R}_{+}^{n}$ and $u \in \mathfrak{M}_{\omega}^{\prime}$ for some $\omega$. We say that $u \in M(\Omega ; \varrho) 2$-locally at $\left(0, \delta x_{0}\right)$ if there exists a conical cut-off function $\kappa$ with $\widetilde{\kappa}\left(\delta x_{0}\right) \neq 0$ and $\kappa u \in M(\Omega ; \varrho)$. As in [4], $R=P-Q, P(z)=R(0, z)$, where $R$ is a singular elliptic operator of order $m$.

Theorem 4 (B. Ziemian). Let $w \in M(\Omega ; s)$ 2-locally at $\left(0 ; \delta x_{0}\right)$. If $u \in \mathfrak{M}_{\alpha}^{\prime}$ satisfies

$$
P u=Q(\kappa u)+\kappa w \quad \text { in } \mathbb{R}_{+}^{n}
$$

then $\kappa u \in M\left(\Omega \backslash \bigcup_{j=0}^{\infty}\left\{\operatorname{Char}_{\widetilde{\alpha}} P+j\right\} ; s-m\right)$, where $j=(j, 0, \ldots, 0) \in \mathbb{N}_{0}^{n}$.

It is worth mentioning that usually solutions to the equation $R u=w$ have continuous radial asymptotics even though the right-hand side has a discrete asymptotic expansion. 
As we see the notion of the radial characteristic set plays an important role in regularity theory of solutions to corner elliptic equations. Below we describe this set for a class of polynomials in $\mathbb{C}^{2}$.

Let $P(z)=\sum_{|\nu|=m} b_{\nu} z^{\nu}$ be a polynomial of order $m$ in two complex variables with constant coefficients and let

$$
\mathcal{P}\left(\zeta_{1}, \zeta_{2}\right)=P\left(\zeta_{1}-\zeta_{2}, \zeta_{2}\right)=a_{m} \prod_{j=1}^{m}\left(\zeta_{2}-c_{j}\left(\zeta_{1}\right)\right)
$$

By assumption $P$ is non-characteristic in $(-1,1)$ and $\mathcal{P}$ has no multiple polynomial factors. Define the discriminant of $\mathcal{P}$ by

$$
\Delta=\prod_{j<k}\left(c_{j}\left(\zeta_{1}\right)-c_{k}\left(\zeta_{1}\right)\right) .
$$

Fix $\widetilde{\alpha} \in \mathbb{R}^{2}$ and let $\widetilde{a}=A \widetilde{\alpha}$. Let $\theta_{\nu}(\nu=1, \ldots, N)$ be all points in $\mathbb{C}$ such that $\Delta\left(\theta_{\nu}\right)=0$. Denote by $B_{\mu}(\mu=1, \ldots, M)$ all points in $\mathbb{R}$ such that for some $j$, $\operatorname{Re} c_{j}\left(\widetilde{a}_{1}+i B_{\mu}\right)=\widetilde{a}_{2}$. Define

$$
\begin{gathered}
L_{\mu}=\mathbb{R}+i B_{\mu}, \quad \widetilde{L}_{\nu}=\overline{\mathbb{R}}_{+}+\theta_{\nu}, \quad Z=\left\{\zeta_{1}: \Delta\left(\zeta_{1}\right)=0\right\}, \\
L=\bigcup_{\mu=1}^{M} L_{\mu} \cup \bigcup_{\nu=1}^{N} \widetilde{L}_{\nu} \cup Z, \quad L_{\widetilde{a}}=L \cap\left\{\operatorname{Re} \zeta_{1} \geq \widetilde{a}_{1}\right\} .
\end{gathered}
$$

In this situation we can write $\operatorname{Char}_{\widetilde{\alpha}} P=A^{-1}\left(L_{\widetilde{a}} \times \mathbb{C}\right)$, and a refinement of the regularity theorem can be proved.

3. Second microlocalization. It is well known that non-linear singularities do not have to propagate along bicharacteristic curves. In other words different non-linear waves may interact producing new singular waves. The most fundamental example here is the case of a semilinear wave operator in two space variables $\square u=f(x, u), x \in \mathbb{R}^{3}$. To study such waves we need more information on the way the solution approaches the singular set. This is achieved by a further localization of solution called the second microlocalization. It was studied by M. Kashiwara, J. M. Bony and others. In the case when the singular set reduces to the origin, second microlocalization consists (roughly speaking) in localizing $u$ (in addition to microlocalizing it) to a conical neighbourhood in the $x$ space of a vector $\delta \widetilde{x}$. The effect is then measured in terms of the scale of suitable weighted Sobolev spaces. For instance one may use the space $S P\left(s, s^{\prime}\right)$.

Let $u \in \mathcal{D}^{\prime}\left(\mathbb{R}^{n} \backslash 0\right), u \equiv 0$ in the unit ball. Let $s+s^{\prime}$ be a non-negative integer. We say that $u \in S P\left(s, s^{\prime}\right)$ if

$\|x\|^{-s+|\lambda|} D^{\lambda} u \in L^{2}\left(\mathbb{R}^{n}\right) \quad$ for $0 \leq|\lambda| \leq s+s^{\prime}, \lambda \in \mathbb{R}^{n},|\lambda|=\lambda_{1}+\ldots+\lambda_{n}$. 
For remaining $\left(s, s^{\prime}\right)$ the spaces $S P\left(s, s^{\prime}\right)$ are defined by duality and interpolation. Fix $\delta \widetilde{x} \in \mathbb{R}_{+}^{n}, \widetilde{\xi} \in \mathbb{R}^{n}$ and let:

$1^{\circ} \varrho \in C^{\infty}\left(i\left(\mathbb{R}^{n} \backslash\{0\}\right)\right)$ be a homogeneous function of order zero defined in a conical neighbourhood of $i \widetilde{\beta}=i \delta \widetilde{x} \widetilde{\xi}, \varrho(i \widetilde{\beta}) \neq 0$. The function $\varrho$ is extended to $\mathbb{R}^{n}+i\left(\mathbb{R}^{n} \backslash\{0\}\right)$ by putting $\varrho(z)=\varrho(\operatorname{Im} z)$.

$2^{\circ} \kappa=\varphi \cdot \kappa^{\prime}$ where $\varphi$ is a $C_{0}^{\infty}$ bump function at zero and $\kappa^{\prime} \in C^{\infty}\left(\mathbb{R}^{n} \backslash\{0\}\right)$ is a cut-off function in a conical neighbourhood of $\delta \widetilde{x}$, i.e., $\operatorname{supp} \kappa^{\prime} \subset \mathbb{R}_{+}^{n}, \kappa^{\prime}$ is homogeneous of order zero and $\kappa^{\prime}(\delta \widetilde{x}) \neq 0$.

$3^{\circ} \chi \in C^{\infty}\left(i\left(\mathbb{R}^{n} \backslash\{0\}\right)\right)$ be a function with supp $\chi \subset\{i \tau: 1 / 4 \leq\|\tau\|\}$ and $\chi(i \tau) \equiv 1$ for $\|\tau\| \geq 1 / 2$. The function $\chi$ is extended to $\mathbb{R}^{n}+i\left(\mathbb{R}^{n} \backslash\{0\}\right)$ by putting $\chi(z)=\chi(\operatorname{Im} z)$.

Definition 1 (J. M. Bony [1]). Let $(0, \xi) \in T_{0}^{*}\left(\mathbb{R}^{n}\right), \delta \widetilde{x} \in \mathbb{R}_{+}^{n}, \delta \widetilde{\xi}=0$. Let $u \in S P(s,-\infty)=\bigcup_{\sigma} S P(s, \sigma)$. We say that $u$ belongs to $S P\left(s, s^{\prime}\right)$ 2-microlocally at $(\widetilde{x}, \widetilde{\xi}, \delta \widetilde{x}, \delta \widetilde{\xi})=(0, \widetilde{\xi}, \delta \widetilde{x}, 0)$ if there exist functions $\varrho, \kappa, \chi$ satisfying conditions $1^{\circ}, 2^{\circ}, 3^{\circ}$ respectively such that

$$
\widetilde{P}(x, D) u=\chi(x D) \varrho(x D) \kappa(x) u \in S P\left(s, s^{\prime}\right) \quad\left(x D=\left(x_{1} D_{1}, \ldots, x_{n} D_{n}\right)\right) .
$$

If $u \in S P(s,-\infty)$ we define its $S P\left(s, s^{\prime}\right)$-second wave front set (denoted by $\left.2 \mathrm{WF}_{S P\left(s, s^{\prime}\right)}\right)$ as a closed subset of the space

$$
\left(T^{*} \mathbb{R}^{n} \backslash T_{0}^{*}\left(\mathbb{R}^{n}\right)\right) \cup N_{T_{0}^{*}\left(\mathbb{R}^{n}\right)} T\left(T^{*} \mathbb{R}^{n}\right)
$$

consisting of the points $(x, \xi) \notin T_{0}^{*}\left(\mathbb{R}^{n}\right)$ such that $u \notin S P\left(s+s^{\prime}, 0\right)$ microlocally at $(x, \xi)$ (observe that outside of zero, $S P\left(s, s^{\prime}\right)=S P\left(s+s^{\prime}, 0\right)$ coincides with the usual Sobolev space) and of the points $(0, \xi, \delta x, 0)$ such that $u \notin S P\left(s, s^{\prime}\right) 2$-microlocally.

This definition however is not practical and one would like to have a definition in terms of growth of a suitable transformation. Clearly the Fourier transformation is not convenient here since the spaces $S P\left(s, s^{\prime}\right)$ are not isotropic. Instead one can use the approach based on the Mellin transformation (Bony uses in this situation complicated techniques of Paley-Littlewood decompositions).

Let $\Gamma \subset \mathbb{R}_{+}^{n}$ be a proper cone. If $u \in S P\left(s, s^{\prime}\right), s+s^{\prime} \in \mathbb{N}_{0}$ and supp $u \subset \Gamma$ then

(i) $\mathcal{M} u \in \mathcal{O}\left(\sum_{j=1}^{n} \operatorname{Re} z_{j}<s-n / 2\right)$.

(ii) $\mathcal{M} u(\alpha+i \cdot) \in L^{2, s+s^{\prime}}\left(\mathbb{R}^{n}\right)=L^{2}\left(\mathbb{R}^{n},(1+\|\beta\|)^{s+s^{\prime}}\right)$ for $\alpha$ such that $\sum \alpha_{j} \leq-n / 2$.

This leads to the following equivalent definition of the second wave front set.

Definition $2([8])$. Let $(0, \widetilde{\xi}) \in T_{0}^{*}\left(\mathbb{R}^{n}\right), \delta \widetilde{x} \in \mathbb{R}_{+}^{n}, u \in S P(s,-\infty)$. Then $u \in S P\left(s, s^{\prime}\right)$ 2-microlocally at $(0, \widetilde{\xi}, \delta \widetilde{x}, 0)$ if there exist functions 
$\varrho, \kappa, \chi$ satisfying $1^{\circ}, 2^{\circ}, 3^{\circ}$ such that

$$
\left.\chi(z) \varrho(z) \mathcal{M}(\kappa u)(z)\right|_{z=\alpha+i} \in L^{2, s+s^{\prime}}\left(\mathbb{R}^{n}\right) \quad \text { for } \alpha_{1}+\ldots+\alpha_{n} \leq s-n / 2 .
$$

Now we can formulate the main result of [8].

Theorem 5 (Propagation of singularities along outgoing bicharacteristics). Let $\delta \widetilde{x}=(1,0), \widetilde{\xi}=\left(0, \widetilde{\xi}^{\prime}\right)$ and let $v \in S P(s,-\infty)$ be such that $v \in S P(s, \sigma) 2$-microlocally at the points $(0, \widetilde{\xi}, \delta x, 0)$ where $\delta x \in \mathbb{R}_{+}^{n}$. Suppose $w=\frac{\partial}{\partial x_{1}} v$ and $w \in S P(s-1, \sigma+1) 2$-microlocally at $(0, \widetilde{\xi}, \delta \widetilde{x}, 0)$. If $\sigma<-1 / 2$ then $v \in \operatorname{SP}(s, \sigma) 2$-microlocally at $(0, \widetilde{\xi}, \delta \widetilde{x}, 0)$.

A similar theorem is true for incoming and second bicharacteristics. These theorems together give the well known Bony theorem on the propagation of 2-microlocal singularities (see [1]).

\section{References}

[1] J. M. Bony, Second microlocalization and propagation of singularities for semilinear hyperbolic equations, in: Hyperbolic Equations and Related Topics (Kataka/Tokyo, 1984), Academic Press, Boston, 1986, 11-49.

[2] Z. Szmydt and B. Ziemian, Multidimensional Mellin transformation and partial differential operators with regular singularity, Bull. Polish Acad. Sci. Math. 35 (1987), 167-180.

[3] - - - Solutions of singular elliptic equations via the Mellin transformation on sets of high order of tangency to the singular lines, ibid. 36 (1988), 521-535.

[4] - - - The Mellin Transformation and Fuchsian Type Partial Differential Equations, Kluwer, Dordrecht, 1992.

[5] - - - Local existence and regularity of solutions of singular elliptic operators on manifolds with corner singularities, J. Differential Equations 83 (1990), 1-25.

[6] B. Ziemian, Elliptic corner operators in spaces with continuous radial asymptotics, I, ibid. 101 (1993), 28-57.

[7] - Elliptic corner operators in spaces with continuous radial asymptotics, II, in: Banach Center Publ. 27, Inst. Math., Polish Acad. Sci., 1992, 555-580.

[8] B. Ziemian and H. Kołakowski, Second microlocalization and the Mellin transformation, Publ. RIMS Kyoto Univ. 26 (1990), 785-801.

Institute of Mathematics

Warsaw University

Banacha 2

02-097 Warszawa, Poland

E-mail: jhk@mimuw.edu.pl 